\title{
Control of Majorana Edge Modes by a g-factor Engineered Nanowire Spin Transistor
}

\author{
Amrit De ${ }^{1, \text { * }}$ and Alexey A. Kovalev ${ }^{1,2}$ \\ ${ }^{1}$ Department of Physics 63 Astronomy, University of California, Riverside, California 92521, USA \\ ${ }^{2}$ Department of Physics \& Astronomy and Nebraska Center for Materials and Nanoscience, \\ University of Nebraska-Lincoln, Lincoln, NE 68588, USA
}

(Dated: May 27, 2022)

\begin{abstract}
We propose the manipulation of Majorana edge states via hybridization and spin currents in a nanowire spin transistor. The spin transistor is based on a heterostructure nanowire comprising of semiconductors with large and small $\mathrm{g}$-factors that form the topological and non-topological regions respectively. The hybridization of bound edge states results in spin currents and $4 \pi$-periodic torques, as a function of the relative magnetic field angle - an effect which is dual to the fractional Josephson effect. We establish relation between torques and spin-currents in the non-topological region where the magnetic field is almost zero and spin is conserved along the spin-orbit field direction. The angular momentum transfer could be detected by sensitive magnetic resonance force microscopy techniques.
\end{abstract}

Keywords: A. Semiconductors; C. Nanowire Quantum Well; D. Majorana Fermions; D. Spin Transistor;

PACS numbers:

\section{INTRODUCTION.}

It is believed that nanowires with strong spin-orbit interactions can realize topologically protected quantum bits (qubits) [1 3] based on Majorana zero energy modes [4 8 . Some of the other proposals to realize topologically protected qubits include schemes based on topological insulators 9, 10, fractional quantum Hall states [11, cold atom systems [12, 13], p-wave superconductors [14] and superfluids in ${ }^{3} \mathrm{He}-\mathrm{B}$ phase [15].

Typically proposals for observing these Majorana zero energy modes are based on quantum tunneling and transport type phenomena [16 21. Some exciting recent proposals for observing these edge modes are based on the unconventional Josephson effect with a $4 \pi$ periodicity 222 25]. A dual effect has also been suggested in which case a torque between magnets exhibits $4 \pi$ periodicity in the field orientations [26, 27.

It is the dual of Josephson effect that can in principle be employed in spintronic devices. In particular, it is important to understand the role of mechanical torques that should inevitably accompany Majorana hybridization due to conservation of angular momentum. It has been predicted that conservation of angular momentum in macrospin molecules can result in quantum entanglement of a tunneling spin with mechanical modes [28, 29. A flow of spin current between two magnets has been demonstrated to induce spin-transfer torque effect [30, 31 and mechanical torques 32, 33, also by conservation of angular momentum. A flux qubit has been shown to decohere due to exchange of angular momentum between the qubit and elastic modes of a solid 34].

\footnotetext{
${ }^{*}$ Corresponding author. Email : amritde@gmail.com
}

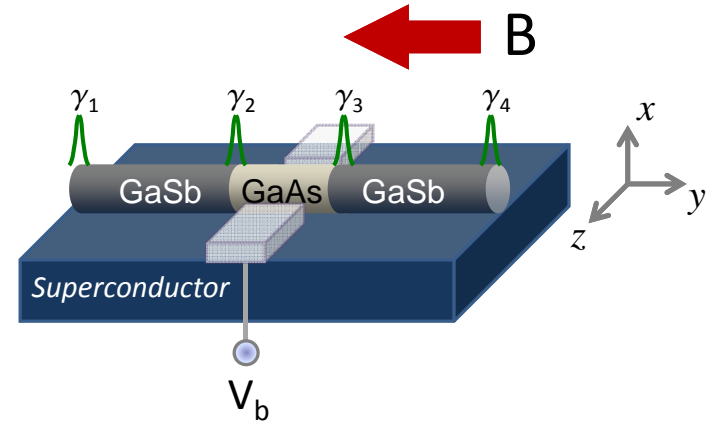

Figure 1: (Color online) A semiconductor nanowire with an embedded type-II GaSb-GaAs-GaSb quantum well placed on top of s-wave superconductor in the presence of a magnetic field. The relatively much smaller g-factor of GaAs makes it a non-topological region. The gate voltage, $V_{b}$, can be used in order to control the hybridization between the edge modes formed at the hetero-junction interface.

In this paper we propose the manipulation of Majorana edge states via hybridization and spin currents using a gated nanowire spin transistor. For our calculations we consider a spin transistor that comprises of GaSbGaAs-GaSb type-II quantum well with strong spin-orbit interactions. Our choice of semiconductors is based on a number of factors. First, the formation of topological and and non-topological region of the wire is determined by the relative $g$-factors of the wires. While GaSb has a large $g \approx-9$, the relatively smaller $g \approx-0.3$ for (GaAs) giving an excellent $g$-factor contrast of about 30. GaSbGaAs nanowires and other Sb containing hetero-junction nanowires have been grown 35 38. Though InSb has a much higher $g$-factor and InSb-InAs heterojunction nanowires have been grown 39 , 40, their $g$-factor contrast is only about 4 . Second, the relatively small con- 
duction band offset of these two materials leads to the formation of a shallow type-II quantum well, thereby only requiring a small gate voltage to raise the chemical potential in the well region.

Typically strong quantum confinement effects can strongly alter the electronic $g$-factors and drive it towards the bare electron value due to effects of orbital angular momentum quenching [41, 42] in materials with sufficiently strong spin-orbit coupling. In the case of InAsInP nanowire quantum dots it has been shown that the $g$-factor can be tuned through zero 43, 44]. Therefore in the case of GaAs (which has a small negative bulk $g$ ), a sufficiently small quantum well should drive $g$ towards 0 , thus making it a perfect non-topological region.

The topological region semiconductor should have strong spin-orbit interactions and large Zeeman splitting. The schematic of our proposal is shown in Fig 1 . The wire is proximity-coupled to an $s$-wave superconductor which results in proximity induced pairing in the wire. The spin transistor allows the bound edge states to hybridize thus resulting in spin-current induced $4 \pi$-periodic torque, as a function of the relative magnetic field angle. As an example of application for our proposal, arrays of nanowires with zero energy edge modes could be used as quantum memory - in which case there arises a need to efficiently read out information from memory elements. The spin and angular momentum flows discussed here could be employed for that. In general the nanowire architecture allows the combination of various lattice mismatched materials and have attracted much attention due to their potential electronic and optoelectronic applications such as single electron transistors, field sensors, and low-power electronics [38, 45, 46.

We also establish relation between torques and spincurrents in the non-topological region where the magnetic field is almost zero and spin is conserved along the spin-orbit field direction. Sensitive magnetic resonance force microscopy measurements can provide further evidence for the existence of these edge modes and their hybridization. Finally, we show that this non-dissipative spin current can be controlled by the external gate voltage (see Fig. 1) which leads to similar functionality with Datta and Das spin-field-effect transistor [47.

\section{TIGHT BINDING CALCULATIONS FOR SPIN CURRENTS AND EDGE HYBRIDIZATION}

Consider a semiconducting quantum wire, with Rashba spin-splitting, placed on top of a superconducting substrate (as per the coordinates shown in Fig. 11). In this solid state system, Majorana fermions are charge less, localized zero-energy collective quasiparticle excitations of the superconducting ground state that satisfy the Bololiubov-de Gennes (BdG) Hamiltonian:

$$
\mathcal{H}=\frac{k^{2}}{2 m} \tau_{z}+i \alpha_{s o} k_{y} \tau_{z} \sigma_{z}+\boldsymbol{\Delta}^{\prime} \cdot \boldsymbol{\tau}+\mathbf{B} \cdot \boldsymbol{\sigma}
$$

where we have used the Nambu spinor basis $\Psi^{T}=$ $\left(\psi_{\uparrow}, \psi_{\downarrow}, \psi_{\downarrow}^{\dagger},-\psi_{\uparrow}^{\dagger}\right), \boldsymbol{\sigma}$ and $\boldsymbol{\tau}$ are Pauli vectors that respectively act on particle and hole sectors. Here $\alpha_{\text {so }}$ is the strength of Rashba spin-splitting term, $\mathbf{B}=$ $\left[B_{o} \cos \theta,-B_{o} \sin \theta,-B_{z}\right]$ is the magnetic field vector, $\boldsymbol{\Delta}^{\prime}=[\Delta \cos \phi, \Delta \sin \phi,-\mu], \mu$ is the chemical potential and $\Delta e^{i \phi}$ is the superconducting pairing potential. In general, the energy spectrum of the BdG Hamiltonian supports gapped and gapless phases. The overall phase diagram is more complicated than TI edge systems [26, 27] due to the presence of the $k^{2} \tau_{z}$ term. Here, we limit our consideration to the $\Delta^{2}>B_{z}^{2}$ part of the phase diagram where the energy bands are always gapped. There are two gaped phases, - topological(T) for $\Delta^{2}-B_{z}^{2}<B_{o}^{2}-\mu^{2}$ and non-topological(N) for $\Delta^{2}-B_{z}^{2}>B_{o}^{2}-\mu^{2}$, separated by a quantum phase transition at $\Delta^{2}-B_{z}^{2}=B_{o}^{2}-\mu^{2}$.

The coupling between a magnetic field and the spin of an electron is determined by the $g$-factor, which would therefore determine whether the semiconductor is in the $N$ or $T$ phase. Hence, it is possible to engineer a nanowire quantum well structure that can form $T|N| T$ or $N|T| N$ regions even when placed in a uniform magnetic field. For a $T|N| T$ type system, the hybridization across the $N$ region (which forms the well) can be gate controlled. Our proposed spin transistor that comprises of GaSbGaAs-GaSb type-II quantum well within a nanowire is shown in Fig. 1 .

In order to treat arbitrary 1D heterostructures and non-uniform magnetic fields, we transform the $\mathrm{BdG}$ Hamiltonian, Eq. (1), onto the following tight binding model with nearest neighbor hopping:

$$
\begin{aligned}
\mathcal{H}= & \sum_{j, \sigma, \sigma^{\prime}}\left[c_{j+1 \sigma}^{\dagger}\left(-t_{0} \sigma_{0}+i \frac{\alpha_{j}}{2} \sigma_{z}\right)_{\sigma \sigma^{\prime}} c_{j \sigma^{\prime}}+H . c .\right] \\
& +\sum_{j, \sigma}\left(2 t_{0}-\mu_{i}\right) c_{j \sigma}^{\dagger} c_{j \sigma}+\sum_{j}\left(\widetilde{\Delta}_{i} c_{j \uparrow}^{\dagger} c_{j \downarrow}^{\dagger}+H . c .\right) \\
& +\sum_{j}\left(\tilde{B}_{j} c_{j \uparrow}^{\dagger} c_{j \downarrow}+H . c .\right)
\end{aligned}
$$

where we have used the complex parameters $\widetilde{\Delta}=$ $\Delta \exp (i \phi), \widetilde{B}=B_{o} \exp (-i \theta)$ and $c_{j \sigma}^{\dagger}\left(c_{j \sigma}\right)$ creates (annihilates) an electron of spin $\sigma$ on site $j$. The proximity induced gap $\Delta=0.5 \mathrm{meV}$. Here $t_{0}=\hbar / 2 \mathrm{~m}^{*} \mathrm{a}^{2}$ is the hopping strength, $\alpha_{j}=\alpha_{s o}^{(j)} / a$ where $a$ is the lattice spacing. In our calculations, we use the following parameters for $\mathrm{GaSb}$ and GaAs, $m_{G a S b}=0.041 m_{e}, m_{G a A s}=0.067 m_{e}$, $\alpha_{s o}^{G a S b}=0.187 \mathrm{eV \AA}$ and $\alpha_{s o}^{G a A s}=0.024 \mathrm{eV} \AA$. The magnetic field at each site is given by $\tilde{B}_{j}=g_{j} \mu_{B} \mathcal{B} / 2$, where $\mu_{B}$ is the Bohr Magneton, $g_{j}$ is the Lande g-factor of the semiconductor at that given lattice site and $\mathcal{B}$ is the applied field. Although quantum confinement effects can alter the electronic $g$-factors in materials with sufficiently strong spin-orbit coupling [41, 42, 44, as we are considering non-topological well regions that are fairly large - we use the bulk $g$-factors: $g_{G a A s}=-0.32$ and $g_{G a S b}=-8.72$. 

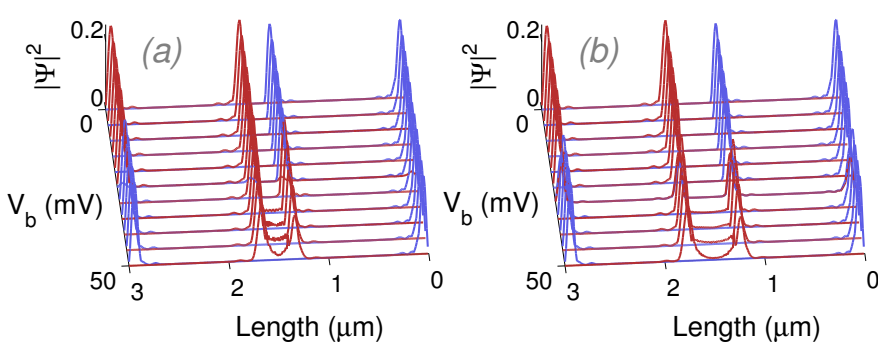

Figure 2: (Color online) Edge modes as a function of heterojunction bias voltage $V_{b}$ with the wire subjected to constant magnetic field for the non-topological GaAs well widths of (a) $200 \mathrm{~nm}$ and (b) $400 \mathrm{~nm}$. Hybridization of the Majorana edge modes is seen at $V_{b}=30 \mathrm{meV}$.

The overall length of the wire was taken to be $3 \mu \mathrm{m}$ corresponding to 300 grid sites for a grid spacing of $a=10 \mathrm{~nm}$. Typically the GaSb section of the nanowires are about $60 \mathrm{~nm}$ in diameter, while the GaAs sections are about $40 \mathrm{~nm}$ wide 36 . Taking the effective masses, the transverse quantization, the bandgaps and the valance band offset of these semiconductors in account, we estimate that the barrier height of the quantum well is about $52 \mathrm{meV}$.

In Fig 2 we show the edge states from our tight binding calculations as a function of the bias voltage applied to the non-topological GaAs well region for two different well widths. The respective energies of these states are shown in Fig 3. It is clearly seen that the shorter non-topological well region results in higher hybridization energies due to more dominant finite size effects. In the absence of any bias, the quantum well prevents the hybridization of the edge modes as indicated by the separate red and blue edge states. This separation of the edge modes persists till the threshold $V_{b} \approx 30 \mathrm{mV}$ is reached at which point a split in the energy spectrum is seen due to hybridization of the edge modes. As $V_{b}$ is further increased, the edge modes abruptly return to their unhybridized state as the bias voltage now acts as a barrier preventing any tunneling effects. The unhybridized Majorana edge modes $\left(\gamma_{1}\right.$ and $\left.\gamma_{4}\right)$ are formed at the ends of the structure and they have nearly zero energy. The hybridized edge modes $\left(\gamma_{2}\right.$ and $\left.\gamma_{3}\right)$ are formed in the middle and they have non-zero energy due to finite size effects. This non-zero energy edge mode can now be manipulated by spin currents and magnetic field gradients.

In general the three components of the spin current operator are as follows

$$
J_{j}(r)=\mathcal{R} e\left(\Psi^{\dagger}(r)\left[\sigma_{j} \frac{\partial \hat{H}}{\partial p}+\frac{\partial \hat{H}}{\partial p} \sigma_{j}\right] \Psi(r)\right)
$$

where $j=x, y, z$ and $\Psi(r)$ is the position dependent Nambu spinor which can be calculated by diagonalizing the tight binding Hamiltonian. The spin currents were numerically calculated using finite differences and the tight binding wavefunctions.

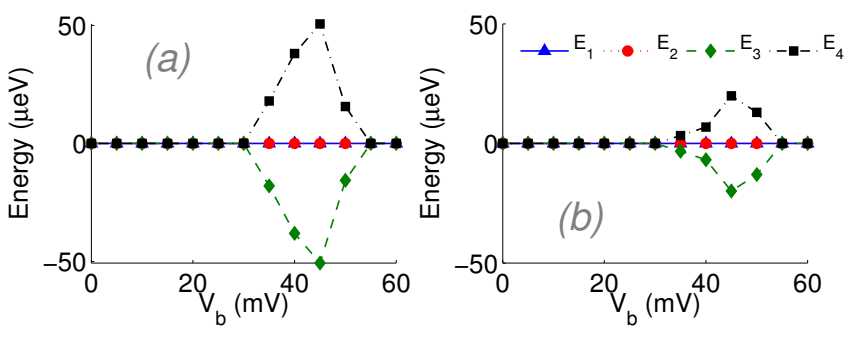

Figure 3: (Color online) The hybridization energies as a function of bias voltage corresponding to Fig 2 for well widths of (a) $20 \mathrm{~nm}$ and (b) $40 \mathrm{~nm}$.

The spatial distribution of the $z$ component of the spin current for the hybridized edge states as a function of various gate bias voltages is shown in Fig, 4 . The Rashba spin-splitting is along the $z$-direction and only the $J_{z}$ spin current is non zero in the non-topological GaAs well region (spin is conserved along the $z$-direction for nontopological region). It is seen that the gate bias voltage can strongly affect the spin current. Overall the spin current is nearly constant in the $N$ region and is therefore conserved.

In Fig 5 (a), we plot hybridization energies of the edge states as a function of the relative angle, $\theta$, between the magnetic fields on the left and right topological GaSb region. Separate magnetic tips with localized dipole type fields could be used to realize this. Notice the $4 \pi$ periodic behavior. The corresponding spin torque $\partial E^{\mathrm{n}}(\theta) / \partial \theta$ agrees with spin current in Fig 5 (b). It is the $\theta$ dependence of the hybridization energy that leads to mechanical torques. Such torques could be detectable by the exertion of a mechanical torque on a nano-magnetic tip [26, 27]. In the presence of hybridization in the topological region we observe difference between the spin current and torque which is the result of additional torques produced by the coupling between the orbital and spin degrees of freedom.

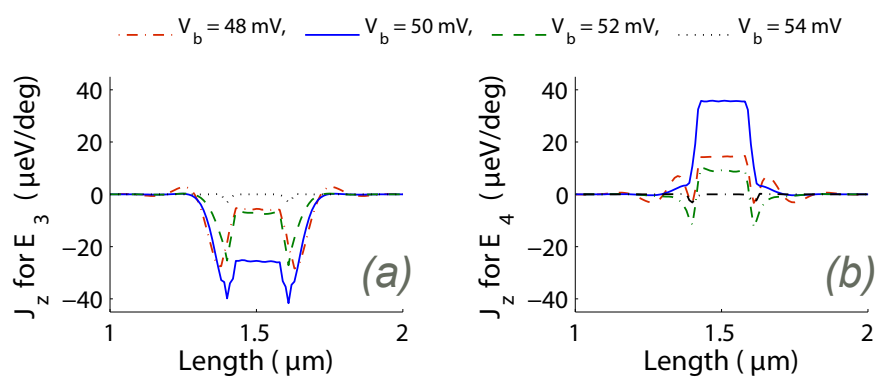

Figure 4: (Color online) The spatial distribution of the $z$ component of the spin current for the (a) $E_{3}$ and (b) $E_{4}$ states as a function of various bias voltages, $V_{b}$. The spin current only exists in the middle section when the Majorana edge modes are allowed to hybridize over the non-topological region. Note that the real part of $x$ and $y$ components of the spin currents is zero. 

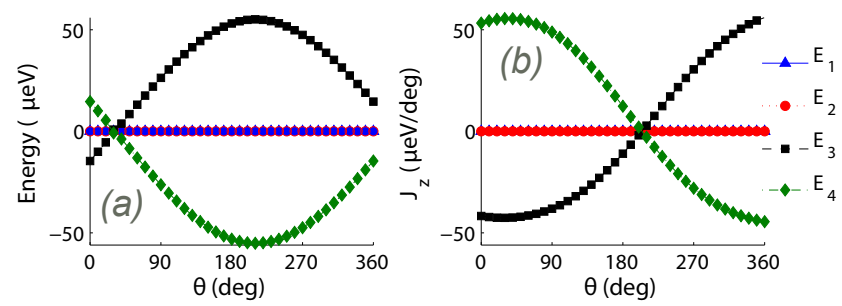

Figure 5: (Color online) (a) Hybridization energy of Majorana bound states as a function of the relative angle, $\theta$, between the magnetic fields on the left and right $T$ region (separate dipolar magnetic tips with more localized fields can be used for this). Note the $4 \pi$ periodic behavior. (b) The spin current as function of $\theta$ with bias voltage of $V_{b}=50 \mathrm{mV}$ applied.

\section{ANALYTIC RESULTS}

The numerical results presented in the previous section can be better understood by the analytical results presented in this section.

In general, an effective two level system or a Majorana qubit can be formed by four Majorana edges where three of the edges are hybridized (in Fig. 11 we assume that edges $\gamma_{1}$ and $\gamma_{2}$ are separated from each other). The effective low energy Hamiltonian can be written as

$$
\mathcal{H}=i E^{\mathrm{n}}(\theta) \gamma_{2} \gamma_{3}+i E^{\mathrm{t}} \gamma_{3} \gamma_{4}
$$

where $\theta$ is the angle between the magnetic fields, $\gamma_{i}$ describe Majorana edge states that can form two fermions $b^{\prime}=\gamma_{1}+i \gamma_{2}$ and $b^{\prime \prime}=\gamma_{3}+i \gamma_{4}$, where $b^{\dagger}(b)$ are ferminoic creation(anhilation) operators.

We analyze the hybridization of the edge modes and resulting spin-currents and torques on $T|N| T$ structures. In order to calculate $E^{\mathrm{n}}(\theta)$, we consider a semiconductor nanowire with two infinite $T$ regions $(\mathrm{GaSb})$ separated by a finite $N$ region (GaAs). We introduce parameters $\left\{\Delta_{L}, B_{L}, \mu_{L}, \theta_{L}\right\}$ for the left $T$ region, $\left\{\Delta_{M}, \mu_{M}\right\}$ for the middle $N$ region and $\left\{\Delta_{R}, B_{R}, \mu_{R}, \theta_{R}\right\}$ for the right $T$ region. The phase of superconducting pairing is assumed constant (i.e. $\phi=0$ ) throughout the wire. In order to determine the bound state at a single $T \mid N$ boundary, we need to find the 4-component zero energy solution to the Hamiltonian (Eq. 11). We use ansatz $\Psi(x)=$ $\exp (\kappa x) \Psi(\kappa)$, where $\kappa$ is complex. In doing so we arrive at four solutions that decay into the topological region, i.e. with $\mathcal{R} e(\kappa)>0$, and four solutions that decay into the non-topological region, i.e. with $\mathcal{R} e(\kappa)<0$. A linear combination of these solutions on each side has to be continuous and have continuous derivative at the $T \mid N$ boundary which leads to unique solution for the edge state.

We denote such solutions as $\left|\psi_{L}\right\rangle=\exp \left[i \theta_{L} \sigma_{z} / 2\right]\left|\psi_{L}^{0}\right\rangle$ for the left Majorana edge and as $\left|\psi_{R}\right\rangle=$ $\exp \left[i \theta_{R} \sigma_{z} / 2\right]\left|\psi_{R}^{0}\right\rangle$ for the right Majorana edge. Next we employ lowest order perturbation theory to find the hybridization energy of Majorana modes and spin current at the boundary when the solutions for the left and right edges weakly overlap. For the hybridization energy we obtain:

$$
E^{\mathrm{n}}(\theta) \approx \frac{\left|\left\langle\psi_{L}|H| \psi_{R}\right\rangle\right|}{\sqrt{\left\langle\psi_{L}^{0} \mid \psi_{L}^{0}\right\rangle\left\langle\psi_{R}^{0} \mid \psi_{R}^{0}\right\rangle}}
$$

which becomes

$$
E^{\mathrm{n}}(\theta) \approx E_{0}^{\mathrm{n}} \exp \left[-\mathcal{R} e\left(\kappa_{2}^{n}\right) \ell_{n}\right] \cos \left[\frac{\theta}{2}+\Phi_{0}+\mathcal{I} m\left(\kappa_{2}^{n}\right) \ell_{n}\right]
$$

where $\kappa_{2}^{n}=m^{*} / \hbar\left(i \alpha_{s o}-i \sqrt{2(i \Delta+\mu) \hbar / m^{*}+\alpha_{s o}^{2}}\right), E_{0}^{\mathrm{n}}$ and $\Phi_{0}$ depend on the parameters of the $T$ and $N$ regions and not on the wire length, $\ell_{n}$, and $\theta$.

Similarly for a semiconductor nanowire with two infinite $N$ regions separated by a finite $T$ region (GaAs), the hybridization energy for the topological region is:

$$
\begin{aligned}
E^{\mathrm{t}} \approx E_{0}^{\mathrm{t}} & \left(\exp \left[-\kappa_{2}^{t} \ell_{t}\right]+\right. \\
& \left.\left|A_{0}\right| \exp \left[-\mathcal{R} e\left(\kappa_{1}^{t}\right) \ell_{t}\right] \cos \left[\arg A_{0}+\mathcal{I} m\left(\kappa_{1}^{t}\right) \ell_{t}\right]\right)
\end{aligned}
$$

where $\kappa_{1}^{t}$ and $\kappa_{2}^{t}$ are the roots of

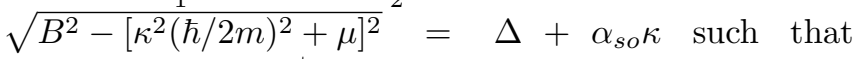
$\Re(\kappa)>0$. Here $E_{0}^{\mathrm{t}}$ and $A_{0}$ depends on parameters of the $T$ and $N$ regions and not on $\ell_{t}$ and $\theta$.

From the perturbative solutions we can express spin current at point $r$ as

$$
\begin{aligned}
& J_{z}(r)= \\
& \mathcal{R} e\left(\frac{\left[\psi_{L}^{\dagger}(r) \pm i \psi_{R}^{\dagger}(r)\right]\left\{\sigma_{z}, \hat{v}\right\}\left[\psi_{L}(r) \pm i \psi_{R}(r)\right]}{2 \sqrt{\left\langle\psi_{L}^{0} \mid \psi_{L}^{0}\right\rangle\left\langle\psi_{R}^{0} \mid \psi_{R}^{0}\right\rangle}}\right)
\end{aligned}
$$

where $\hat{v}=\partial \hat{H} / \partial p$ and $\theta=\theta_{R}-\theta_{L}$. This leads to:

$$
J_{z}= \pm \frac{\partial E^{\mathrm{n}}(\theta)}{\partial \theta},
$$

which relates spin current to the hybridization energy over the $N$ region. This shows that the torque $\partial E^{\mathrm{n}}(\theta) / \partial \theta$ is generated solely by the spin current passing through the middle $N$ region.

\section{MANIPULATION OF MAJORANA QUBIT BY GATES}

Without loss of generality we can fix the electron parity, e.g. 1. The available Hilbert space of two fermions corresponding to four edges $\left(b^{\prime}=\gamma_{1}+i \gamma_{2}\right.$ and $b^{\prime \prime}=$ $\left.\gamma_{3}+i \gamma_{4}\right)$ is $\alpha|1,0\rangle+\beta|0,1\rangle$. This is equivalent to a Hilbert space of a spin $1 / 2$ system. The Hamiltonian in Eq. (4) then becomes:

$$
\mathcal{H}=\frac{E^{\mathrm{n}}(\theta)}{4} \sigma_{x}+\frac{E^{\mathrm{t}}}{4} \sigma_{z},
$$


which shows that by gate tuning $E^{\mathrm{n}}$ and $E^{\mathrm{t}}$ one can perform arbitrary rotations of the Majorana qubit. The relative angle between magnetic fields, $\theta$, can be also used for manipulations. Form the analysis in the previous section it becomes clear that rotations along the $x$-axis are accompanied by mechanical torques. Nevertheless, we estimate that this should not be a strong source of decoherence [34.

\section{SUMMARY}

We have proposed the manipulation of Majorana edge states in a gated nanowire spin transistor that comprises of GaSb-GaAs-GaSb type-II quantum well. The formation of topological and and non-topological region of the wire is determined by the large $g$-factor contrast of the two semiconductors. In general larger spin-orbit interactions (coupled with smaller band gaps) lead to larger g-factors. It is possible to obtain a full Bloch sphere rotations of the Majorana qubit by gates controlling the hybridization energies. The setup can be easily generalized to a larger number interchanging topologi$\mathrm{cal} /$ nontopological regions where universal rotations can be achieved by applying time dependent gates. Spin currents and torques can be further used in order to couple such a wire to various read out schemes 48. The flows of angular momentum, comprising a signature of Majorana edge states, can be detected by sensitive magnetic resonance force microscopy techniques.
[1] V. Mourik, K. Zuo, S. M. Frolov, S. R. Plissard, E. P. A. M. Bakkers, and L. P. Kouwenhoven, Science 336, 1003 (2012).

[2] M. T. Deng, C. L. Yu, G. Y. Huang, M. Larsson, P. Caroff, and H. Q. Xu, Nano Letters 12, 6414 (2012).

[3] L. P. Rokhinson, X. Liu, and J. K. Furdyna, Nat Phys 8, 795 (2012).

[4] E. Majorana, Nuovo Cimento 14, 171 (1937).

[5] F. Wilczek, Nature Phys. 5, 614 (2009).

[6] R. F. Service, Science 332, 193 (2011).

[7] C. Beenakker, Annu. Rev. Condens. Matter Phys. 4, 113 (2013).

[8] J. Alicea, Rep. Prog. Phys. 75, 076501 (2012).

[9] L. Fu and C. Kane, Phys. Rev. Lett. 100, 096407 (2008).

[10] J. Nilsson, A. R. Akhmerov, and C. W. J. Beenakker, Phys. Rev. Lett. 101, 120403 (2008).

[11] G. Moore and N. Read, Nucl. Phys. B 360, 362 (1991), ISSN 0550-3213.

[12] V. Gurarie, L. Radzihovsky, and A. V. Andreev, Phys. Rev. Lett. 94, 230403 (2005).

[13] L. Jiang, T. Kitagawa, J. Alicea, A. R. Akhmerov, D. Pekker, G. Refael, J. I. Cirac, E. Demler, M. D. Lukin, and P. Zoller, Phys. Rev. Lett. 106, 220402 (2011).

[14] T. M. Rice and M. Sigrist, J. Phys. Condens. Matter 7, L643 (1995).

[15] M. Silaev and G. Volovik, J. Low Temp. Phys. 161, 460 (2010), ISSN 0022-2291.

[16] D. Bagrets and A. Altland, Phys. Rev. Lett. 109, 227005 (2012).

[17] J. Liu, A. C. Potter, K. T. Law, and P. A. Lee, Phys. Rev. Lett. 109, 267002 (2012).

[18] D. I. Pikulin, J. P. Dahlhaus, M. Wimmer, H. Schomerus, and C. W. J. Beenakker, New Journal of Physics 14, 125011 (2012).

[19] E. J. H. Lee, X. Jiang, R. Aguado, G. Katsaros, C. M. Lieber, and S. De Franceschi, Phys. Rev. Lett. 109, 186802 (2012).

[20] S. Das Sarma, J. D. Sau, and T. D. Stanescu, Phys. Rev. B 86, 220506 (2012).

[21] A. D. K. Finck, D. J. Van Harlingen, P. K. Mohseni, K. Jung, and X. Li, Phys. Rev. Lett. 110, 126406 (2013).

[22] A. Y. Kitaev, Physics-Uspekhi 44, 131 (2001).

[23] L. Fu and C. L. Kane, Phys. Rev. B 79, 161408 (2009).
[24] R. M. Lutchyn, J. D. Sau, and S. Das Sarma, Phys. Rev. Lett. 105, 077001 (2010).

[25] L. Jiang, D. Pekker, J. Alicea, G. Refael, Y. Oreg, and F. von Oppen, Phys. Rev. Lett. 107, 236401 (2011).

[26] Q. Meng, V. Shivamoggi, T. L. Hughes, M. J. Gilbert, and S. Vishveshwara, Phys. Rev. B 86, 165110 (2012).

[27] L. Jiang, D. Pekker, J. Alicea, G. Refael, Y. Oreg, A. Brataas, and F. von Oppen, Phys. Rev. B 87, 075438 (2013).

[28] A. A. Kovalev, L. X. Hayden, G. E. W. Bauer, and Y. Tserkovnyak, Phys. Rev. Lett. 106, 147203 (2011).

[29] D. A. Garanin and E. M. Chudnovsky, Phys. Rev. X 1, 011005 (2011).

[30] L. Berger, Phys. Rev. B 54, 9353 (1996).

[31] J. Slonczewski, Journal of Magnetism and Magnetic Materials 159, L1 (1996).

[32] A. A. Kovalev, G. E. W. Bauer, and A. Brataas, Phys. Rev. B 75, 014430 (2007).

[33] G. Zolfagharkhani, A. Gaidarzhy, P. Degiovanni, S. Kettemann, P. Fulde, and P. Mohanty, Nat Nano 3, 720 (2008), ISSN 1748-3387.

[34] E. Chudnovsky, D. Garanin, and M. O'Keeffe, Journal of Superconductivity and Novel Magnetism 25, 1007 (2012), ISSN 1557-1939.

[35] Y. N. Guo, J. Zou, M. Paladugu, H. Wang, Q. Gao, H. H. Tan, and C. Jagadish, Applied Physics Letters 89, 231917 (pages 3) (2006).

[36] B. Ganjipour, H. A. Nilsson, B. M. Borg, L.-E. Wernersson, L. Samuelson, H. Q. Xu, and C. Thelander, Applied Physics Letters 99, 262104 (pages 3) (2011).

[37] D. L. Dheeraj, G. Patriarche, H. Zhou, T. B. Hoang, A. F. Moses, S. GrÃ ,nsberg, A. T. J. van Helvoort, B.-O. Fimland, and H. Weman, Nano Letters 8, 4459 (2008).

[38] B. M. Borg and L.-E. Wernersson, Nanotechnology 24, 202001 (2013).

[39] D. Ercolani, F. Rossi, A. Li, S. Roddaro, V. Grillo, G. Salviati, F. Beltram, and L. Sorba, Nanotechnology 20, 505605 (2009).

[40] A. Pitanti, D. Ercolani, L. Sorba, S. Roddaro, F. Beltram, L. Nasi, G. Salviati, and A. Tredicucci, Phys. Rev. X 1, 011006 (2011).

[41] C. E. Pryor and M. E. Flatté, Phys. Rev. Lett. 96, 026804 (pages 4) (2006). 
[42] A. De, C. E. Pryor, and M. E. Flatté, Phys. Rev. Lett. 102, 017603 (2009).

[43] M. T. Björk, A. Fuhrer, A. Hansen, M. Larsson, L. E. Froberg, and L. Samuelson, Physical Review B 72, 201307 (2005).

[44] A. De and C. E. Pryor, Physical Review B 76, 155321 (2007).

[45] C. Thelander, T. M. rtensson, M. T. Björk, B. J. Ohlsson, M. W. Larsson, L. R. Wallenberg, and L. Samuelson,
Appl. Phys. Lett. 83, 2052 (2003).

[46] L. Samuelson, C. Thelander, M. T. Björk, M. Borgström, K. Deppert, K. A. Dick, A. E. Hansen, T. Mårtensson, N. Panev, A. I. Persson, et al., Physica E 25, 313 (2004). [47] S. Datta and B. Das, Appl. Phys. Lett. 56, 665 (1990).

[48] A. A. Kovalev, A. De, and K. Shtengel, arXiv 1306.2339 (2013). 